\title{
The effects of training procedure, response availability, and response meaningfulness in multiple-choice paired-associate learning'
}

\begin{abstract}
The present study investigated the effectiveness of prompting and confirmation training procedures in a multiple-choice paired-associate task in which response availability and response meaningfulness also were manipulated. There were no overall differences in learning due to the training variable, but several significant interaction effects were found which indicated that the effectiveness of a particular training procedure may depend upon the nature of the learning materials.
\end{abstract}

Problem

Several studies have shown that a "prompting" training procedure (i.e., the $\mathrm{S}$ is prohibited from making erroneous responses) is more effective than a "confirmation" procedure (i.e., trial-and-error responding) in the learning of paired-associates (e.g.. Cook, 1958; Cook \& Spitzer, 1960). However, the present writer, using a multiple-choice pairedassociate task, obtained only partial support for these results (Hawker, 1964). In this study it was found that while prompting did have a facilitating effect during the early training trials, there was no overall superiority of either procedure in terms of total number of correct responses or the number of items correctly reproduced in a post-session free-recall test. Hence, it was felt that additional information is needed to determine more precisely how the two training procedures operate in multiple-choice pairedassociate learning, and how they interact with various task variables. The use of a multiple-choice technique permits one to manipulate the nature of the response alternatives in several ways, and the present study was directed toward examining the effects of manipulating response availability and response meaningfulness. Response availability is here defined as representing the total number of response terms available to the $\mathrm{S}$, while response meaningfulness is used in a general sense to refer to whether adjectives or nonsense line figures are used as response terms.

Method

A 2 by 2 by 2 factorial design was employed in which the three variables were training procedure (prompting or confirmation), total number of response terms available ( 8 or 16), and method of presentation (figure-word or word-figure). Six Ss, undergraduate psychology students, were randomly assigned to each of the eight experimental conditions, and each $\mathrm{S}$ was run individually.
All Ss learned the same list of eight pairs of adjectives and nonsense line-figures (list " $\mathrm{A}$ " in the earlier study); however, the order of the pairs was dependent upon the method of presentation-i.e., figureword $(\mathrm{F}-\mathrm{W})$ or word-figure $(\mathrm{W}-\mathrm{F})$. The learning materials were presented on the self-paced memory drum described in the previous study, and using a multiplechoice technique, each presentation consisted of a stimulus term and four response terms. In the eight response term condition, only the eight "legitimate" response terms in the basic list were used in making up alternatives, while in the other condition, the eight legitimate responses plus eight additional irrelevant but comparable response terms were used in making up alternatives. The alternative responses in both conditions were arranged such that, over all trials, each response term appeared an equal number of times as an alternative, and the particular alternatives for a given stimulus term varied from trial to trial. Eight different orders of presentation were used to preclude any serial learning.

The experimental procedure was the same as that described in detail in the earlier study. All Ss were given seven series of practice and test trials to learn the list; each series consisted of two practice trials following the prescribed training procedure and a test trial during which the $\mathrm{S}$ received no knowledge of results. If the $\mathrm{S}$ registered two consecutive errorless test trials, the experiment was concluded and the scores on the last test trial were recorded as the data for the remaining test trials. In addition, the $\mathrm{S}$ was given a free-recall test $5 \mathrm{~min}$. after the end of the experiment proper in which he was required to reproduce the correct response term for each stimulus term.

\section{Results and Discussion}

The mean number of correct responses per group on each of the seven test trials is shown in Fig. 1. Analysis of the total number of correct responses indicated no significant main effects, but there was a significant interaction between response availability and method of presentation, $F(1,40)=5.32, p<.05$. This interaction indicated that with adjectives as response terms (method F-W), increasing the total number of available response terms increased the number of correct responses, but with nonsense figures as response terms (method W-F), increasing the number of response terms decreased the number of correct responses. This interaction suggests that 


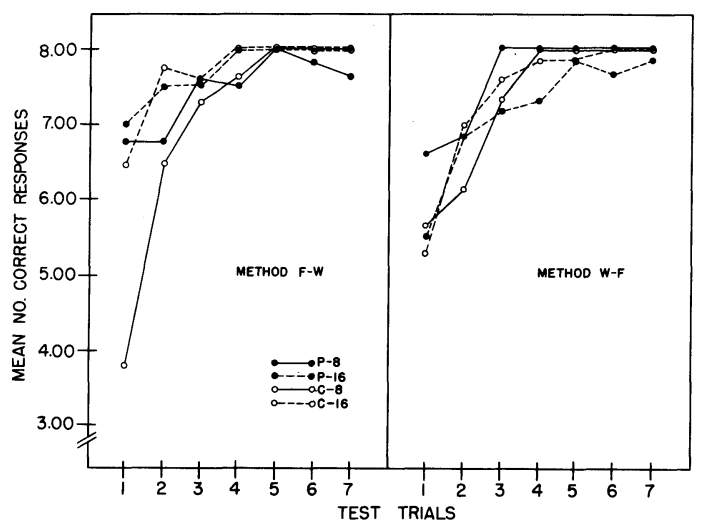

Fig. 1. Mean number of correct responses on each test trial.

when the response terms have high meaningfulness, increasing the size of the available response pool facilitates learning by making the discrimination among response alternatives easier; with low meaningful responses, however, the response terms are not as well-differentiated and hence increasing the number of terms used in making up alternatives provides more elements which may cause interference. Somewhat similar results were reported by Epstein \& Streib (1962) who found, using a multiple-choice technique, that a list in which irrelevant response terms were used as alternatives took significantly fewer trials to learn than a list in which only the "legitimate" response terms were used as alternatives.

A second analysis was performed by combining the scores on the first three test trials and comparing them with the combined scores on the last three test trials. This analysis indicated a significant interaction effect between training procedure and trial blocks, $F(1,40)=4.13, p<.05$, which showed that prompting did produce more correct responses than confirmation during the first block of trials, but that the two procedures were equally effective during the last block of trials, and corroborates the results previously reported by Hawker (1964).

Since most of the Ss (94\%) reached a mastery criterion of two consecutive erroriess test trials.

\section{TABLE I}

Total Trials to Criterion and Free-Recall Scores

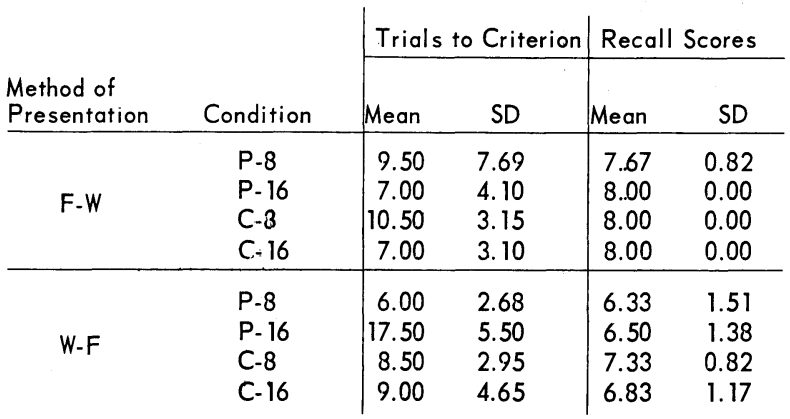

total trials to criterion were computed and the group data are presented in Table 1 (for those Ss who failed to reach criterion, a score of 24-i.e., eight series-was arbitrarily given). Analysis of these scores again indicated no significant main effects, but the response availability by method of presentation interaction was again significant, $F(1,40)=11.89, p<.01$, and lends further support to the Epstein \& Streib finding. Moreover, there was a significant interaction between training procedure and response availability, $F(1,40)=5.29$, $\mathrm{p}<.05$, which showed that increasing the number of response terms significantly increased the number of trials required to reach criterion by Ss using a prompting procedure, but produced a negligible effect for Ss using a confirmation procedure. This differential effect was especially noticeable when the nonsense figures were the response terms (see Table 1) and suggests that the interaction varied as a function of response meaningfulness (i.e., method of presentation). There was, in fact, some evidence for such an interaction, but the associated $\mathrm{F}$-value was not sufficiently large, $\mathrm{F}(1,40)=3.67, \mathrm{p}<.10$.

Two final comparisons dealt with the time required to complete each test trial, and the number of items correctly reproduced in the post-session free-recall test. The time scores were analyzed by comparing performance speed over trial blocks and indicated only a significant trials effect. The previous study had shown that prompting resulted in generally faster performance speeds than did confirmation, and while a similar trend was seen in the present data, the effect was not near significance $F(1,40)=2.03, p<.25$. Analysis of the free-recall scores (see Table 1) also failed to reveal any differences between prompting and confirmation $(F<1.00)$, but did indicate that more word than figure responses were correctly recalled, $F(1,40)=22.68, p<.001$.

The present results, then, are in agreement with those previously reported and again indicate that while prompting does have a facilitating effect during the early training trials, it is not generally any more effective than a confirmation procedure, at least in multiple-choice paired-associate learning. In fact, the interaction with response availability noted in the analysis of trials to criterion suggests that the effectiveness of a particular training procedure may be dependent upon the nature of the learning materials.

\section{References}

Cook, J. O. Supplementary report: Processes underlying learning a single paired-associate item. J. exp. Psychol., 1958, 56, 455.

Cook, J. O., \& Spitzer, M. E. Supplementary report: Prompting versus confirmation in paired-associate learning. J. exp. Psychol., $1960,59,275-276$.

Epstein, W., \& Streib, R. The effect of stimulus meaningfulness and response meaningfulness in the absence of response learning. $J$. verbal Learn. verbal Behav., 1962, 1, 105-108.

Hawker, J. R. The influence of training procedure and other task variables in paired-associate learning. J. verbal Learn. verbal Behav., 1964, 3, 70-76.

Note

1. Portions of this research were presented at the Southwestern Psychological Association meeting in Dallas, April, 1963. 